T. Fujita

Nagoya Math. J.

Vol. 115 (1989), 105-123

\title{
REMARKS ON QUASI-POLARIZED VARIETIES
}

\author{
TAKAO FUJITA
}

Dedicated to Professor Morikawa on his 60th birthday

\section{Introduction}

Let $V$ be a variety, which means, an irreducible reduced projective scheme over an algebraically closed field $\mathcal{\Omega}$ of any characteristic. A line bundle $L$ on $V$ is said to be nef if $L C \geqq 0$ for any curve $C$ in $V$. Thus, "nef" is never an abbreviation of "numerically equivalent to an effective divisor". $L$ is said to be big if $\kappa(L)=n=\operatorname{dim} V$. In case $L$ is nef, it is big if and only if $L^{n}>0$ (cf. [F7; (6.5)]. When $L$ is nef and big, the pair $(V, L)$ will be called a quasi-polarized variety.

We have $\chi(V, t L)=\sum_{j=0}^{n} \chi_{j} t^{[j]} / j$ ! for some integers $\chi_{0}, \chi_{1}, \cdots, \chi_{n}$ where $t^{[j]}=t(t+1) \cdots(t+j-1)$ and $t^{[0]}=1$. By Riemann-Roch Theorem we have $\chi_{n}=L^{n}$. Moreover, if $V$ is normal, we have $-2 \chi_{n-1}=(\omega+(n-1) L) L^{n-1}$ for a canonical divisor $\omega$ of $V$. We set $g(V, L)=1-\chi_{n-1}$, which is called the sectional genus of $(V, L)$. We set $\Delta(V, L)=n+L^{n}-h^{0}(V, L)$, which is called the $\Delta$-genus of $(V, L)$. We expect that we can describe the structure of $(V, L)$ if $\Delta$ and/or $g$ are small enough. When $L$ is ample, we have the results in [F5], [F10], which we will generalize in this paper. Most results were announced in [F11].

In $\S 1$ we show $\Delta \geqq 0$ for any quasi-polarized variety $(V, L)$, and describe the structure of $(V, L)$ with $\Delta=0$ precisely. In particular $g=0$ in this case. We conjecture the converse:

ConJeCture. $g \geqq 0$ for any quasi-polarized variety. Moreover, $g=0$ implies $\Delta=0$ if $V$ is normal.

This is completely unknown when $\operatorname{char}(\Re)>0$, even if $V$ is nonsingular and $L$ is ample. So, from $\S 2$ on, we assume $\operatorname{char}(\Re)=0$. In $\S 2$ we give characterizations of $\boldsymbol{P}^{n}$ and hyperquadrics, which establish

Received July 22, 1988. 
our Conjecture in case $L^{n} \leqq 2$. In $\S 3$ we consider the case in which $L$ is ample. We improve a few results in [F10], allowing $V$ to have certain non-Gorenstein singularities. In $\S 4$ we show that our Conjecture follows from the Flip Conjecture in [KMM]. Hence it is true when $n \leqq 3$ by virtue of Mori's result [M2]. In $\S 5$ we consider the case in which $L$ is spanned by global sections. We give a couple of generalizations of Sommese's result in [S2].

Suitable generalized versions of Kodaira's vanishing theorem, due to Kawamata, Viehweg et al., play an important role in our method. Hironaka's desingularization theory is also indispensable. Nevertheless it is very likely that our Conjecture is true in positive characteristic cases too.

\section{Notation and Convention}

We use the notation in [F10], which is based on the customary one in algebraic geometry. In particular, given a morphism $X \rightarrow Y$ and a line bundle $L$ on $Y$, we denote $f^{*} L$ by $L_{X}$, or sometimes just by $L$ when confusion is impossible or harmless.

\section{$\S 1$. Quasi-polarized varieties of $\Delta$-genus zero}

(1.1) The main result of this section is the following

TheOREM. $\quad \Delta(V, L) \geqq 0$ for any quasi-polarized variety $(V, L)$. Moreover, if $\Delta=0$, there exist a variety $W$, a birational morphism $f: V \rightarrow W$ and $a$ very ample line bundle $H$ on $W$ such that $L=f^{*} H$ and $\Delta(W, H)=0$.

(1.2) Definition. An element of $\operatorname{Pic}(V) \otimes \boldsymbol{Q}$ will be called a $\boldsymbol{Q}$ bundle on a variety $V$. The tensor product in $\operatorname{Pic}(V)$ gives the addition and will be denoted additively. Multiplicative notation will be used for intersection products in Chow rings. We define $\boldsymbol{Q}$-valued intersection numbers of $\boldsymbol{Q}$-bundles in the natural way. A $\boldsymbol{Q}$-bundle $B$ is said to be nef if $B C \geqq 0$ for any curve $C$ in $V$.

As for $L$-dimension of Iitaka, we have $\kappa(m L)=\kappa(L)$ for any positive integer $m$ and $L \in \operatorname{Pic}(V)$. Therefore $\kappa(B)$ is well-defined for any $\boldsymbol{Q}$-bundle $B$ and we have $\kappa(m B)=\kappa(B)$ for any $m>0 . \quad B$ is said to be big if $\kappa(B)=\operatorname{dim} V . \quad B$ is said to be ample if $m B=A$ in $\operatorname{Pic}(V) \otimes \boldsymbol{Q}$ for some $m>0$ and an ample line bundle $A$.

Let $\operatorname{Div}(V)$ (resp. $C_{n-1}(V)$ ) be the group of Cartier (resp. Weil) divisors 
on $V$. An element of $\operatorname{Div}(V) \otimes \boldsymbol{Q}$ (resp. $C_{n-1}(V) \otimes \boldsymbol{Q}$ ) will be called a $\boldsymbol{Q}$-divisor (resp. $\boldsymbol{Q}$-Weil-divisor) on $V$. For any $\boldsymbol{Q}$-Weil-divisor $Z$, we have $Z=\sum m_{i} Z_{i}$ for some $m_{i} \in \boldsymbol{Q}$ and prime Weil divisors $Z_{i} . \quad Z$ is said to be effective if $m_{i} \geqq 0$ for every $i$. In the obvious way a $\boldsymbol{Q}$-divisor $D$ defines a $\boldsymbol{Q}$-bundle, which will be denoted by $[D]$, or just by $D$ by abuse of notation. $D$ is said to be nef (resp. big, etc.) if so is $[D]$.

(1.3) Kodaina's Lemma. A $\boldsymbol{Q}$-bundle $B$ is big if and only if $B-D$ is ample for some effective $\boldsymbol{Q}$-divisor $D$.

For a proof, see, e.g., [F4, (2.8)].

(1.4) Proposition. Let $L$ be a nef big $Q$-bundle on a variety $V$ and let $f: V \rightarrow S$ be a surjective morphism. Let $F$ be a general fiber of $f$. Then the restriction of $L$ to any prime component of $F$ is nef big.

Proof. The nefness is obvious. To prove the bigness, take an effective $Q$-divisor $D$ such that $A=L-D$ is ample. Since $F$ is general, $D$ is effective on any component $X$ of $F$. Since $A_{X}=L_{X}-D_{X}$ is ample, $L_{X}$ is big.

(1.5) Now we prove the theorem (1.1). We may assume $\Delta(V, L)=$ $n+L^{n}-h^{0}(V, L) \leqq 0$, so $h^{0}(V, L)>n$. Let $G$ be the normalization of the graph of the rational mapping defined by $|L|$. Then we have a birational morphism $\pi: G \rightarrow V$, a morphism $\rho: G \rightarrow P \simeq \boldsymbol{P}^{N}$ with $N=h^{0}(V, L)$ -1 and an effective Cartier divisor $E$ on $G$ such that $\pi^{*}|L|=E+\rho^{*}|H|$ for $H=\mathcal{O}_{P}(1)$. Set $W=\rho(G), k=\operatorname{dim} W$ and $w=\operatorname{deg} W$, and let $F$ be a general fiber of $\rho$. Then $0 \leqq \Delta\left(W, H_{W}\right) \leqq k+w-(N+1)$. Moreover $\operatorname{dim} F=n-k$ and $L^{n-k}\{F\}>0$ by (1.4). So $L^{n-k} H^{k}=w L^{n-k} F \geqq w$ on $G$. Since $L$ and $H$ are nef and $E=L_{G}-H_{G}$ is effective, we have $L^{n} \geqq$ $L^{n-k} H^{k}$. Combining these inequalities we get $n+L^{n} \geqq n+w \geqq N+1$ $=h^{0}(V, L)$, which proves $\Delta(V, L) \geqq 0$.

If $\Delta=0$, we must have equalities. Hence $\Delta(W, H)=0, k=n, \rho$ is birational and $L^{n}=H^{n}$ on $G$. We will derive a contradiction assuming $E \neq 0$.

Since $L^{n}=L^{j} H^{n-j}=H^{n}$ for any $j$, we have $H^{n-1} E=0$. This implies $\operatorname{dim} \rho(E)<n-1$. Therefore, by a similar argument as in $[\mathrm{F} 8 ;(1.5)]$ using index theorem, we infer that there is a curve $C$ such that $E C<0$ and $\rho(C)$ is a point. Then $L C=(E+H) C<0$, contradicting the nefness of $L$. 
Thus we see $E=0$. So $L$ is spanned by global sections and $|L|$ gives a morphism $f: V \rightarrow W$ having the desired properties.

(1.6) Remark. We have a complete classification theory of polarized varieties with $\Delta=0$ (see [F1], [F5]). In particular $W$ is normal and locally Cohen-Macaulay, and $g(W, H)=0$.

(1.7) Corollary. $L$ is spanned by global sections and $g(V, L)=0$ for any quasi-polarized variety $(V, L)$ with $\Delta(V, L)=0$.

Proof. The spannedness is clear since $H$ is very ample. By (1.6), $g(V, L)=0$ follows from the lemma below.

(1.8) Lemma. Let $f: V \rightarrow W$ be a birational morphism onto a normal variety $W$. Then $g(W, H)=g\left(V, f^{*} H\right)$ for any line bundle $H$ on $W$.

Proof. We have $f_{*} \mathcal{O}_{V}=\mathcal{O}_{W}$ since $W$ is normal. Moreover, since $f$ is birational, $\operatorname{dim}\left(\operatorname{Supp}\left(\mathscr{F}_{q}\right)\right)<n-1$ for any $q>0$, where $\mathscr{F}_{q}=R^{q} f_{*} \mathcal{O}_{V}$. Hence $\chi(V, t L)=\sum(-1)^{q} \chi\left(W, \mathscr{F}_{q}(t H)\right) \sim \chi(W, t H)$ modulo terms of degree $<n-1$ in $t$. So $\chi_{n-1}(V, L)=\chi_{n-1}(W, H)$ and the assertion follows.

\section{$\S 2$. Characterizations of projective spaces and hyperquadrics}

From now on, throughout this paper, we assume $\operatorname{char}(\Re)=0$.

(2.1) Theorem. Let $L$ be a line bundle on a smooth variety $M$ and let $K$ be the canonical bundle. Suppose that $L-(K+D)$ is nef and big for some negligible $Q$-divisor $D$. Then $H^{q}(M, L)=0$ for any $q>0$.

Here, $D=\sum m_{i} D_{i}$ is said to be negligible if $\operatorname{Supp}(D)=\cup D_{i}$ has no singularity other than normal crossings and if $0 \leqq m_{i}<1$ for every $i$. For a proof of this theorem, see [K], [V].

The two theorems below are the main results of this section.

(2.2) Theorem. Let $(V, L)$ be a normal quasi-polarized variety and suppose that $H^{n}\left(M,-\pi^{*} t L\right)=0$ for $1 \leqq t \leqq n=\operatorname{dim} V$, where $\pi: M \rightarrow V$ is a desingularization of $V$. Then there is a birational morphism $f: V \rightarrow P$ $\simeq \boldsymbol{P}^{n}$ with $L=f^{*} \mathcal{O}_{P}(1)$.

(2.3) Theorem. Let $(V, L)$ be a normal quasi-polarized variety and suppose that $h^{n}\left(M,-\pi^{*} t L\right)=0$ for $0<t<n=\operatorname{dim} V$ and $h^{n}(M,-n L)=1$ for a desingularization $\pi: M \rightarrow V$. Then either

1) $L^{n}=g(V, L)=1$, or 
2) there is a birational morphism $f: V \rightarrow Q$ onto a (possibly singular) hyperquadric $Q$ in $P^{n+1}$ such that $L=f^{*} \mathcal{O}_{Q}(1)$.

Remark. Case 1) does really occur when $(V, L)$ is a scroll over an elliptic curve.

(2.4) In order to prove these theorems, we need a few lemmas.

Lemma. Let $A$ and $B$ be nef $\boldsymbol{Q}$-bundles on a variety $V$ and set $I_{a}=$ $A^{a} B^{n-a} \in \boldsymbol{Q}$ for $a=0,1, \cdots, n$. Then $I_{a-1} I_{a+1} \leqq I_{a}^{2}$ for any $0<a<n$.

It is well-known that this follows from the index theorem on surfaces. See, e.g., [F6; $(1.2: 4)]$.

(2.5) Lemma. Let $L$ be a nef big $Q$-bundle on a smooth variety $M$ with $n=\operatorname{dim} M$ and let $E$ be an effective $Q$-divisor on $M$ such that $L^{n-1} E$ $=0$. Suppose that $L+E-F$ is nef for some effective $\boldsymbol{Q}$-divisor $\boldsymbol{F}$. Then $F-E$ is effective.

Proof. By (1.3), there is an effective $Q$-divisor $D$ such that $L-D$ is ample. Then $L-\varepsilon D$ is ample for any $\varepsilon$ with $0<\varepsilon \leqq 1$ since $L$ is nef. We will derive a contradiction assuming that $F-E$ is not effective.

Take a small enough $\varepsilon>0$ such that $F+\varepsilon D-E$ is not effective and set $A=(L-\varepsilon D)+(L+E-F)$, which is an ample $\boldsymbol{Q}$-bundle on $M$. By virtue of Hironaka's theory we can find a birational morphism $\pi: M_{1} \rightarrow M$ and effective $Q$-divisors $E_{1}, F_{1}$ and $E_{0}$ on $M_{1}$ such that $\pi^{*} E=$ $E_{0}+E_{1}, \pi^{*}(F+\varepsilon D)=E_{0}+F_{1}$ and $E_{1} \cap F_{1}=\varnothing$. Note that $\pi_{*} E_{1} \neq 0$ by assumption.

We claim that $B=F_{1}+A_{1}$ is nef for $A_{1}=\pi^{*} A$. To see this, note that $B=L_{1}+E_{1}$, where $L_{1}=2 \cdot \pi^{*} L$. Now, for any curve $C$ in $M_{1}$, we have $E_{1} C \geqq 0$ or $F_{1} C \geqq 0$ since $E_{1} \cap F_{1}=\varnothing$. Hence $B C \geqq 0$ because both $L_{1}$ and $A_{1}$ are nef.

Since $L_{1}$ is nef and $L_{1}^{n-1} \pi^{*} E=0$, we have $L_{1}^{n-1} Y=0$ for any component $Y$ of $\pi^{*} E$. In particular $L_{1}^{n-1} E_{1}=0$. Therefore $B L_{1}^{n-1}=L_{1}^{n}$. This implies $B^{n} \leqq B^{n-1} L_{1} \leqq \cdots \leqq B L_{1}^{n-1}=L_{1}^{n}$ by (2.4). On the other hand $B^{n}=B^{n-1}\left(L_{1}+E_{1}\right) \geqq B^{n-1} L_{1}$. Hence $B^{n-1} E_{1}=0$. So $A_{1}^{n-1} E_{1}=0$ since $B=$ $F_{1}+A_{1}$ and $E_{1} \cap F_{1}=\varnothing$. But $A_{1}^{n-1} E_{1}=A^{n-1} \pi_{*} E_{1}>0$ since $A$ is ample. Thus we get a contradiction, as desired.

(2.6) Corollary. Let $L$ be a nef big line bundle and let $E$ be an effective divisor on $M$ such that $L^{n-1} E=0$. Then $H^{0}(M, L) \simeq H^{0}(M, L+E)$. 
Proof. Take a birational model $\pi ; M_{1} \rightarrow M$ such that $\pi^{*}|L+E|=$ $F+\Lambda$ for some effective divisor $F$ and a linear system $\Lambda$ with $\operatorname{Bs} \Lambda=\varnothing$. Then $F-\pi^{*} E$ is effective by (2.5). Hence $H^{\circ}\left(M_{1}, \mathcal{O}(\Lambda)\right) \simeq H^{0}\left(M_{1}, \pi^{*} L\right) \simeq$ $H^{0}\left(M_{1}, \pi^{*}(L+E)\right)$.

(2.7) Lemma. Let $L$ be a nef big $Q$-bundle on a variety $V$ and let $N$ be a nef $\mathbf{Q}$-bundle with $L^{n-1} N=0$. Then $N$ is numerically equivalent to zero.

Proof. Take an effective $Q$-divisor $D$ such that $A=L-D$ is ample. Then $L^{n-1} N=L^{n-2}(A+D) N \geqq L^{n-2} A N \geqq \cdots \geqq A^{n-1} N$ since $N$ is nef. So $A^{n-1} N=0$, and [F7; (3.3)] applies.

(2.8) Corollary. Let $L$ be a nef big $Q$-bundle on a variety $V$ and let $E$ be an effective Cartier divisor such that $L^{n-1} E=0 . \quad$ Then $h^{0}(V, \mathcal{O}(E))$ $=1$.

Proof. We will derive a contradiction assuming $\operatorname{dim}|E|>0$. Let $G$ be the graph of the rational mapping defined by $|E|$. Then we have a birational morphism $\pi: G \rightarrow V$ and an effective divisor $F$ on $G$ such that $\pi^{*}|E|=F+H$ for some linear system $H$ with $\operatorname{Bs} H=\varnothing$. Since $L$ is nef and $0=L^{n-1} E=L^{n-1}(F+H) \geqq L^{n-1} H$, we have $L^{n-1} H=0$. So $H$ is numerically trivial by (2.7). This is impossible if $\operatorname{dim}|E|>0$.

Remark. (2.7) and (2.8) are valid in positive characteristic cases too.

(2.9) Now we prove the theorem (2.2). Let $\pi: M \rightarrow V$ be a desingularization and let $K$ be the canonical bundle of $M$. For the sake of brevity $\pi^{*} L$ will be denoted just by $L$. Then we have $h^{q}(M,-t L)=$ $h^{n-q}(M, K+t L)=0$ for $q<n, t>0$. Hence $\chi(t)=\chi(M, t L)=0$ for $-1 \geqq t \geqq-n$. So $\chi(t)=d(t+1) \cdots(t+n) / n$ ! for some constant $d$. By Riemann-Roch Theorem we obtain $d=L^{n}$ and $K L^{n-1}=-d(n+1)$. Since $h^{0}(M, K+(n+1) L)=h^{n}(M,-(n+1) L)=(-1)^{n} \chi(-n-1)=d>0$, we have a member $E$ of $|K+(n+1) L|$. Then $L^{n-1} E=0$. So $d=1$ by (2.8). Moreover, by (2.6), we have $h^{0}(M, L)=h^{0}(M, L+E)=(-1)^{n} \chi(-n-2)=$ $n+1$. Hence $\Delta(M, L)=0$ and $\pi^{*} L$ is spanned by (1.1). So $L$ is spanned on $V$ too since $V$ is normal. Thus $|L|$ gives a mapping with the desired property.

(2.10) Next we prove (2.3) by a similar method. This time we have $h^{n}(M,-t L)=0$ for $1 \leqq t<n$ and hence $\chi(t)=\chi(M, t L)=(t+1) \ldots$ 
$(t+n-1)(d t+a) / n$ ! for some constants $d$, a. Here $d=L^{n}$ and $K L^{n-1}$ $=d(1-n)-2 a / n$. Moreover $\chi(-n)=(-1)^{n}$ since $h^{n}(M,-n L)=1$. So $a=(d-1) n$ and $(K+n L) L^{n-1}=2-d$. Since $h^{0}(M, K+n L)=h^{n}(M,-n L)$ $=1$, we have a member $E$ of $|K+n L|$. Then $L^{n-1} E=2-d \geqq 0$, so $d=1$ or 2 .

When $d=1$, we have $(K+(n-1) L) L^{n-1}=0$. Therefore $g(V, L)=$ $g\left(M, \pi^{*} L\right)=1$ by (1.8). Thus we are in case 1$)$.

When $d=2$, we have $L^{n-1} E=0$. So $h^{0}(M, L)=h^{0}(M, L+E)=$ $(-1)^{n} \chi(-n-1)=n+2$ by $(2.6)$. Hence $\Delta(M, L)=0$. So we are in case 2$)$ by the same reasoning as in (2.9).

(2.11) CoRollary. Let $(V, L)$ be a normal quasi-polarized variety with $g(V, L) \leqq 0, L^{n} \leqq 2, n=\operatorname{dim} V$. Then $\Delta(V, L)=0$.

Proof. Let $\pi: M \rightarrow V$ be a desingularization. Then $\Delta(V, L)=\Delta(M, L)$ and $g(M, L)=g(V, L) \leqq 0$ by (1.8), where $L$ stands for $\pi^{*} L$. So $(K+$ $(n-1) L) L^{n-1} \leqq-2$. If $L^{n}=1$, then $h^{n}(M,-t L)=h^{0}(M, K+t L)=0$ for $t \leqq n$. Hence (2.2) applies.

If $L^{n}=2$, then $h^{n}(M,-t L)=0$ for $t \leqq n$. Moreover $h^{n}(M,-n L)=$ $h^{0}(M, K+n L) \leqq 1$ by (2.8). So (2.3) applies.

(2.12) By a similar method as above we obtain the following

Proposition. Let $(V, L)$ be a quasi-polarized variety of dimension $n$ and assume $H^{q}(V, t L)=0$ for any $q>0,1-n \leqq t \leqq 1$. Then $\Delta(V, L)=0$.

Proof. Setting $\chi(t)=\chi(V, t L)$, we have $\chi(-t)=0$ for $1 \leqq t \leqq n-1$ and $\chi(0)=1$. From this we infer $\chi(t)=(t+1) \cdots(t+n-1)(d t+n) / n$ ! for $d=L^{n}$. Hence $h^{0}(V, L)=\chi(1)=n+d$. Thus $\Delta(V, L)=0$.

(2.13) Remark. Let $(V, L)$ be a quasi-polarized variety such that $h^{n}(V,-t L)=0$ for $1 \leqq t \leqq n$. Then (2.2) applies.

This follows from the lemma below. We have a similar result in case (2.3) too.

(2.14) Lemma. Let $\pi: X \rightarrow V$ be a birational morphism of varieties of dimension $n$. Then $h^{n}(X, \mathscr{F}) \leqq h^{n}\left(V, \pi_{*} \mathscr{F}\right)$ for any coherent sheaf $\mathscr{F}$ on $X$ and $h^{n}\left(X, \pi^{*} \mathscr{E}\right) \leqq h^{n}(V, \mathscr{E})$ for any locally free sheaf $\mathscr{E}$ on $V$.

Proof. Set $\mathscr{F}_{q}=R^{q} \pi_{*} \mathscr{F}$ and let $S_{q}$ be its support. Then $\operatorname{dim} S_{q}<$ $n-q$ for any $q>0$ since $\operatorname{dim} \pi^{-1}\left(S_{q}\right)<n$ and $\operatorname{dim} \pi^{-1}(x) \geqq q$ for any $x \in S_{q}$. So $H^{n-q}\left(V, \mathscr{F}_{q}\right)=0$ for $q>0$. From this we infer $h^{n}(X, \mathscr{F}) \leqq$ 
$h^{n}\left(V, \mathscr{F}_{0}\right)$ using Leray spectral sequence. As for the second assertion, note that the natural homomorphism $\mathscr{E} \rightarrow \pi_{*} \pi^{*} \mathscr{E}$ is injective and $\operatorname{dim}(\operatorname{Supp}(\mathscr{C}))<n$ for its cokernel $\mathscr{C}$. So $h^{n}(V, \mathscr{E}) \geqq h^{n}\left(V, \pi_{*} \pi^{*} \mathscr{E}\right)$. Together with the former inequality this proves the second assertion.

\section{§ 3. Polarized varieties having log-terminal singularities}

(3.1) We review theories in [KMM] used in this paper.

Let $B=\sum b_{i} B_{i}$ be a $\boldsymbol{Q}$-Weil-divisor on a normal variety $V$ with $0 \leqq b_{i}<1$ for each $i$. A birational morphism $\pi: M \rightarrow V$ is called a nice desingularization of the pair $(V, B)$ if $M$ is smooth and if $\pi^{-1}(B \cup S)$ is a divisor having no singularity other than simple normal crossings, where $S=\left\{x \in V \mid \operatorname{dim} \pi^{-1}(x)>0\right\}$.

$(V, B)$ is said to have only log-terminal singularities if there are a nice desingularization as above and effective $Q$-divisors $E, D$ on $M$ such that $\pi_{*} E=0, E$ is a usual divisor, $\pi_{*} D=B, D$ is negligible and $K+D$ $=\pi^{*} \omega(V, B)+E$ for some $\boldsymbol{Q}$-bundle $\omega(V, B)$ on $V$, where $K$ is the canonical bundle of $M$. In this case $\omega(V, B)$ will be called the canonical $\boldsymbol{Q}$ bundle of $(V, B)$.

If $B=0$, we say that $V$ has only log-terminal singularities. In this case $\omega(V, 0)$ corresponds to the canonical sheaf of $V$.

Remark. Log-terminal singularities are rational and they form a wider class than that of canonical singularities in the sense of Reid. Any quogient singularity by a finite group action is log-terminal. A Gorenstein singularity is log-terminal (in fact canonical) if it is rational.

(3.2) Combining [KMM; 1-2-5] and the argument [S2; (0.2.1)], we obtain the following

LEMma. Let $F$ be a finite subset of a variety $V$ and set $U=V-F$. Let $B$ be a $\boldsymbol{Q}$-divisor on $V$ such that $\left(U, B_{U}\right)$ has only log-terminal singularities. Suppcse that $\omega\left(U, B_{U}\right)$ is the restriction of some $\mathbf{Q}$-bundle $\omega$ on $V$ and that $L-\omega$ is nef and big for some line bundle $L$ on $V$. Then $H^{q}(V, L)=0$ for $q>0$.

Proof. Let $\pi: M \rightarrow V$ be a nice desingularization of $(V, B)$ and let $B^{\prime}$ be the proper transform of $B$ on $M$. Then $K+B^{\prime}=\pi^{*} \omega+\sum a_{i} E_{i}$ for some prime divisors $E_{i}$ with $\pi_{*} E_{i}=0$, and $a_{i}>-1$ unless $\pi\left(E_{i}\right) \subset F$. Hence there are effective divisors $P, N$ and a negligible $Q$-divisor $D$ on 
$M$ such that $\pi_{*} P=0, \pi(N) \subset F, \pi_{*} D=B$ and $K+D=\pi^{*} \omega+E$ for $E=P-N$. Then, by (2.1), we have $H^{q}\left(M, \pi^{*} L+E\right)=0$ for $q>0$.

Similarly $H^{q}\left(M, \pi^{*}(L+t H)+E\right)=0$ for $q>0, t \geqq 0$ and any ample line bundle $H$ on $V$. Letting $t \gg 0$ and using Leray spectral sequence, we infer $h^{0}\left(V, R^{q} \pi_{*} \mathcal{O}_{M}\left(\pi^{*}(L+t H)+E\right)\right)=0$, which implies $R^{q} \pi_{*} \mathcal{O}_{M}(L+E)$ $=0$ since $H$ is ample. Using Leray spectral sequence again, we get $H^{q}\left(V, \pi_{*} \mathcal{O}_{M}(L+E)\right)=0$ for $q>0$.

In view of the exact sequence $0 \rightarrow \mathcal{O}_{M}(L+E) \rightarrow \mathcal{O}_{M}(L+P) \rightarrow \mathcal{O}_{N}(L+P)$ $\rightarrow 0$, we infer that $\operatorname{Coker}\left(\pi_{*} \mathcal{O}_{M}(L+E) \rightarrow \pi_{*} \mathcal{O}_{M}(L+P)\right)$ is supported in $\pi(N)=F$. Hence $h^{q}(V, L)=h^{q}\left(V, \pi_{*} \mathcal{O}_{M}(L+P)\right) \leqq h^{q}\left(V, \pi_{*} \mathcal{O}_{M}(L+E)\right)=0$ for $q>0$. Thus we complete the proof.

(3.3) Definition. A pair $(V, L)$ of a variety $V$ and a line bundle $L$ on it is called a scroll over a variety $W$ if there is a vector bundle $\mathscr{E}$ on $W$ such that $V \simeq \boldsymbol{P}(\mathscr{E})$ and $L=\mathcal{O}(1)$. Thus $V$ is a $\boldsymbol{P}^{r-1}$-bundle over $W$ for $r=\operatorname{rank}(\mathscr{E})$.

(3.4) Now we state the main result of this section.

Theorem. Let $(V, L)$ be a polarized variety with $\operatorname{dim} V=n$ and let $B$ be a $\boldsymbol{Q}$-divisor such that $(V, B)$ has only log-terminal singularities. Suppose that there is a subset $Y$ of $V$ such that codim $Y>2$ and $\omega(V, B)_{U}$ comes from $\operatorname{Pic}(U)$ for $U=V-Y$. Then $\omega(V, B)+(n-1) L$ is nef unless $\Delta(V, L)=0$ or $(V, L)$ is a scroll over a smooth curve.

This is proved similarly as [F10; Theorem 2]. The following lemma plays a key role.

(3.5) Lemma. Let $V, L, B$ be as above and suppose that $\omega+m L$ is not nef for $\omega=\omega(V, B)$ and some $m \geqq 0$. Then there is a surjective morphism $f: V \rightarrow W$ onto a normal variety $W$ and a curve $R$ in $V$ with the following properties:

1) Every fiber of $f$ is connected.

2) A curve $C$ in $V$ is numerically proportional to $R$ if and only if $f(C)$ is a point.

3) $F \in \operatorname{Pic}(V)$ comes from Pic $(W)$ if and only if $F R=0$.

4) $F$ is relatively $f$-ample if and only if $F R>0$.

5) $(\omega+m L) R<0$.

This follows from the Cone Theorem in [KMM]. Such a curve $R$ will be called an extremal curve and $f$ will be called the contraction morphism of $R$. 
(3.6) Now we prove (3.4). Suppose that $\omega+(n-1) L$ is not nef. Take $f, W, R$ as in (3.5) with $m=n-1$. We will first prove $\operatorname{dim} W<n$.

Assume that $f$ is birational. Take a point $x$ on $W$ such that $\operatorname{dim} f^{-1}(x)$ $=k>0$. Let $X$ be an irreducible component of $f^{-1}(x)$ with $\operatorname{dim} X=k$, let $\nu: N \rightarrow X$ be its normalization and let $M$ be a smooth model of $N$. For any ample line bundle $H$ on $W$ we have $H^{q}\left(V,-t L+l H_{V}\right)=0$ for any $q>0, t \leqq n-1$ and $l \gg 0$ by (3.2). Hence, similarly as in [F10; (2.3) \& (2.4)], we get $H^{k}\left(M,-t L_{M}\right)=0$ for $t \leqq n-1$. By (2.2) this implies $k=n-1, L_{N}^{k}=1, \Delta\left(N, L_{N}\right)=0$ and hence $\left(N, L_{N}\right) \simeq\left(\boldsymbol{P}^{n-1}, \mathcal{O}(1)\right)$ since $L_{N}$ is ample. Take a general line $Z$ in $N$. Then $\nu(Z) \cap Y=\varnothing$ since $\operatorname{dim}\left(\nu^{-1}(Y)\right)<n-2$. So $\omega Z \in Z$ and $\omega Z \leqq-n$. Hence $-n L-\omega$ is $f$-nef by $(3.5 ; 4)$, which implies $H^{q}\left(V,-n L+l H_{V}\right)=0$ for $q>0, l \gg 0$ by (3.2). So $H^{k}\left(N,-n L_{N}\right)=0$, which is absurd.

(3.7) Proof of (3.4), continued. Now we have $\operatorname{dim} W<n$. Let $F$ be a general fiber of $f$, let $\pi: M \rightarrow F$ be its desingularization and set $k=$ $\operatorname{dim} F$. Similarly as above we get $H^{k}\left(M,-t L_{M}\right)=0$ for any $t \leqq n-1$. So $k \geqq n-1$.

If $k=n-1$, we obtain $\left(F, L_{F}\right) \simeq\left(\boldsymbol{P}^{k}, \mathcal{O}(1)\right)$ by (2.2), since any general fiber is normal by Bertini's theorem. $W$ is a curve in this case. So every fiber $X$ of $f$ is a Cartier divisor with $L^{n-1} X=L^{n-1} F=1$. Hence $X$ is irreducible and reduced since $L$ is ample. By the lower-semicontinuity of the $\Delta$-genus we infer $\left(X, L_{X}\right) \simeq\left(\boldsymbol{P}^{k}, \mathcal{O}(1)\right)$. Thus $(V, L)$ is a scroll over $W$.

When $k=n, W$ is a point. So $\omega$ is numerically equivalent to $c L$ for some rational number $c$ with $c<1-n$. Therefore $H^{q}(V, t L)=0$ for any $q>0, t \geqq 1-n$ by (3.2). So $\Delta(V, L)=0$ by (2.12). Thus we complete the proof of (3.4).

(3.8) Corollary. Let $L$ be an ample line bundle on a log-terminal variety $V$. Suppose that $g(V, L) \leqq 0$ and $V-Y$ is Gorenstein for a subset $Y$ with codim $Y>2$. Then $\Delta(V, L)=0$.

Proof. Set $\omega=\omega(V, 0)$. By (1.8) we have $(\omega+(n-1) L) L^{n-1}=$ $=2 g(V, L)-2<0$ for $n=\operatorname{dim} V$. So $\omega+(n-1) L$ is not nef. By (3.4) we may assume that $(V, L)$ is a scroll over a curve $W$. Then $g(V, L)$ is the genus of $W$ and the assertion follows.

(3.9) Corollary. Let things be as above except $g(V, L)=1$. Then 
$\omega=(1-n) L$ (hence $V$ is Gorenstein) and $\Delta(V, L)=1$, unless $(V, L)$ is a scroll over a smooth elliptic curve.

Proof. By (3.4) we may assume that $F=\omega+(n-1) L$ is nef. Moreover $F L^{n-1}=2 g-2=0$. So $F$ is numerically trivial by (2.7). Now we use (3.2) to get $\chi(V,-t L)=0$ for $1 \leqq t<n-1$ and $\chi(V, \mathcal{O})=1$. From them and $g=1$ we infer $\chi(V, t L)=(t+1) \cdots(t+n-2)\left(d t^{2}+(n-1) d t\right.$ $+(n-1) n) / n !$. Hence $h^{0}(V, L)=\chi(V, L)=n+d-1$ by (3.2). So $\Delta(V, L)$ $=1$. Moreover $\chi(V,(1-n) L)=(-1)^{n}$ implies $h^{0}(V, \omega+(n-1) L)=1$ by duality and (3.2). Hence $\omega=(1-n) L$, as asserted.

\section{§4. Three dimensional cases}

In this section we will show that our Conjecture follows from the Flip Conjecture. In particular, by virtue of Mori's result [M2], our Conjecture is true in dimension $\leqq 3$.

(4.1) Definition. Two quasi-polarized varieties $\left(V_{1}, L_{1}\right)$ and $\left(V_{2}, L_{2}\right)$ are said to be birationally equivalent if there is another variety $G$ with birational morphisms $f_{i}: G \rightarrow V_{i}(i=1,2)$ such that $f_{1}^{*} L_{1}=f_{2}^{*} L_{2}$.

Now we state the main result in this section.

(4.2) Theorem. Let $(V, L)$ be a quasi-polarized variety with $n=$ $\operatorname{dim} V \leqq 3$. Then there exists a quasi-polarized variety $\left(V^{\prime}, L^{\prime}\right)$ which is birationally equivalent to $(V, L)$, has only $\mathbf{Q}$-factorial terminal singularities, and satisfies one of the following conditions.

1) $\omega^{\prime}+(n-1) L^{\prime}$ is nef for the canonical $Q$-bundle $\omega^{\prime}$ of $V^{\prime}$.

2) $\Delta\left(V^{\prime}, L^{\prime}\right)=0$.

3) $\left(V^{\prime}, L^{\prime}\right)$ is a scroll over a curve.

Here, " $\boldsymbol{Q}$-factorial" means that every Weil divisor is $\boldsymbol{Q}$-Cartier. As for the definition of terminal singularities, see, e.g., [KMM]. We remark that they are much milder singularities than log-terminal ones. In particular they are smooth in codimension two.

We make a brief review of theories in [KMM] which we use.

(4.3) Let $V$ be an $n$-dimensional variety having only terminal singularities. If the canonical $Q$-bundle $\omega$ is not nef, then there is an extremal curve $R$ and the contraction morphism $f: V \rightarrow W$ of $R$ as in (3.5).

When $f$ is birational, set $S=\left\{x \in V \mid \operatorname{dim} f^{-1}(x)>0\right\}$ and $E=f^{-1}(S)$. 
$f$ will be called a divisorial (resp. flipping) contraction if $\operatorname{dim} E=n-1$ (resp. $<n-1)$.

(4.4) Lemma. If $f$ is a divisorial contraction as above and if $V$ has only $\boldsymbol{Q}$-factorial terminal singularities, then $W$ has only $\boldsymbol{Q}$-factorial terminal singularities.

For a proof, see [KMM; 5-1-6].

(4.5) FLIP CONJECTURE I. If $f$ is a flipping contraction, there exists a birational morphism $f^{+}: V^{+} \rightarrow W$ from a variety $V^{+}$having only $Q$ factorial terminal singularities such that

1) $f^{+}$is an isomorphism in codimension one, and

2) the canonical $\mathbf{Q}$-bundle $\omega^{+}$of $V^{+}$is $f^{+}$-ample.

This is in fact true when $n \leqq 3$ (cf. [M2]). $f^{+}$is determined uniquely if exists, and is called the flip of $f . V^{+}$is called the flip-flop of $V$ with respect to $R$.

A sequence of varieties $\left\{V_{j}\right\}$ is called a flip-flop sequence if, for each $i, V_{j+1}$ is the flip-flop of $V_{j}$ with respect to an extremal curve $R_{j}$ on $V_{j}$.

(4.6) Flip CONJECTURE II. There does not exist a flip-flop sequence of infinite length.

This is true when $n \leqq 4$ (cf. [Sh] and [KMM; 5-1-15]).

(4.7) Now we prove (4.2). Let $\pi: V_{0} \rightarrow V$ be a desingularization of $V$ and set $L_{0}=\pi^{*} L$. If $\omega_{0}+(n-1) L_{0}$ is nef for the canonical bundle $\omega_{0}$ of $V_{0}$, then we finish by setting $\left(V^{\prime}, L^{\prime}\right)=\left(V_{0}, L_{0}\right)$. So we may assume that $\omega_{0}+(n-1) L_{0}$ is not nef. Take an extremal curve $R_{0}$ with $\left(\omega_{0}+\right.$ $\left.(n-1) L_{0}\right) R_{0}<0$ and let $f_{0}: V_{0} \rightarrow W$ be the contraction morphism of it.

Suppose that $f_{0}$ is not birational. If $L_{0} R_{0}=0$, then $L_{0}$ comes from Pic $(W)$, but this contradicts (1.4). Hence $L_{0} R_{0}>0$ and $L_{0}$ is relatively $f_{0}$-ample. Therefore, similarly as in (3.7), we infer that $\Delta\left(V_{0}, L_{0}\right)=0$ or $\left(V_{0}, L_{0}\right)$ is a scroll over a curve. So we finish by setting $\left(V^{\prime}, L^{\prime}\right)=$ $\left(V_{0}, L_{0}\right)$.

Suppose that $f_{0}$ is birational. If $L_{0} R_{0}>0$, we get a contradiction as in (3.6). So $L_{0} R_{0}=0$ and $L_{0}=f_{0}^{*} L_{W}$ for some line bundle $L_{W}$ on $W$. If $f_{0}$ is a divisorial contraction, we set $\left(V_{1}, L_{1}\right)=\left(W, L_{W}\right)$. If $f_{0}$ is a flipping contraction, we take the flip $f^{+}: V^{+} \rightarrow W$ of $f_{0}$ and $\operatorname{set}\left(V_{1}, L_{1}\right)=\left(V^{+},\left(f^{+}\right)^{*} L_{W}\right)$. In either case $V_{1}$ has only $\boldsymbol{Q}$-factorial terminal singularities and hence 
is smooth in codimension two. Clearly $\left(V_{1}, L_{1}\right)$ is birationally equivalent to $(V, L)$.

We repeat the above argument replacing $\left(V_{0}, L_{0}\right)$ by $\left(V_{1}, L_{1}\right)$. Then we can finish by setting $\left(V^{\prime}, L^{\prime}\right)=\left(V_{1}, L_{1}\right)$, or we get another pair $\left(V_{2}, L_{2}\right)$. In this case we again apply the above argument to $\left(V_{2}, L_{2}\right)$. Thus, we continue until we finish.

We obtain $\left(V_{j+1}, L_{j+1}\right)$ either by a divisorial contraction or by a flipflop as in (4.5). By (4.6), we must have a divisorial contraction after finite steps. Then the Picard number of $V_{j}$ decreases by one, while it does not change in case of flip-flop. Since the Picard number is finite, such a process cannot continue infinitely. Thus we can find $\left(V^{\prime}, L^{\prime}\right)$ with the desired property after finite steps.

(4.8) Corollary. Let $L$ be a nef big line bundle on a normal variety $V$ of dimension $n \leqq 3$. Then $g(V, L) \geqq 0$. Moreover, if $g(V, L)=0$, then $\Delta(V, L)=0$.

Proof. Take $\left(V^{\prime}, L^{\prime}\right)$ as in (4.2) and use the argument in (3.8). Note that $g(V, L)=g\left(V^{\prime}, L^{\prime}\right)$ by $(1.8)$ and $\Delta(V, L)=\Delta\left(V^{\prime}, L^{\prime}\right)$ by the normality of $V$.

(4.9) Corollary. Let $(V, L)$ be as above and suppose $g(V, L)=1$. Then the pair $\left(V^{\prime}, L^{\prime}\right)$ as in (4.2) is either a scroll over an elliptic curve or a locally Gorenstein variety with $\omega^{\prime}=(1-n) L^{\prime}$ and $\Delta\left(V^{\prime}, L^{\prime}\right)=1$.

For a proof, use the argument in (3.9).

\section{§5. The case $L$ is spanned by global sections}

(5.2) When $\mathcal{O}(L)$ is spanned by global sections, our conjecture is easily verified. Indeed, taking general members of $|L|$ successively, we get a sequence $V=V_{n} \supset V_{n-1} \supset \cdots \supset V_{1}$ of subvarieties $V_{j}$ of $V$ such that $V_{j}$ is a member of $\left|L_{V_{j}}\right|$. Then $g(V, L)$ is equal to the arithmetic genus of the curve $V_{1}$ and hence non-negative. Moreover, by Bertini theorem, $V_{j}$ 's are normal if so is $V$. Thus, the following theorem applies if $g=0$.

(5.2) Theorem. Let $(V, L)$ be a quasi-polarized variety with $g(V, L)$ $=0$. Suppose that there is a sequence $\left\{V_{j}\right\}$ as above such that each $V_{j}$ is normal. Then $\Delta(V, L)=0$. 
Proof. We will show $\Delta\left(V_{j}, L\right)=0$ by induction on $j$. This is clear for $j=1$. When $j>1$, we infer $h^{1}\left(V_{j},-L\right)=0$ from (2.1) by Mumford's argument. On the other hand $\Delta\left(V_{j-1}, L\right)=0$ by the induction hypothesis. So $h^{1}\left(V_{j-1}, \mathcal{O}\right)=0$ by $(1.1)$ and by the classification theory of polarized varieties of 4 -genus zero. Combining them we get $h^{1}\left(V_{j}, \mathcal{O}\right)=0$. This implies $\Delta\left(V_{j}, L\right)=\Delta\left(V_{j-1}, L\right)$. Thus we complete the proof.

Remark. If we have a sequence $V=V_{n} \supset \ldots \supset V_{d}$ down to dimension $d$ and if $\Delta\left(V_{d}, L\right)=0$, then we obtain $\Delta(V, L)=0$ by the above argument. In particular, if $d=3$, this method works by virtue of (4.8).

(5.3) Before proceeding further, we make the following remark.

When $L$ is assumed to be spanned by global sections, it is not bad to assume further that $L$ is ample. Indeed, the rational mapping defined by $|L|$ is a morphism in this case. Taking the Stein factorization of it, we get a birational morphism $f: V \rightarrow W$ onto a variety $W$ such that $L=f^{*} A$ for some ample line bundle $A$ on $W$. Thus $(V, L)$ is birationally equivalent to $(W, A)$, and its structure is described via $(W, A)$. We may assume that $W$ is normal if so is $V$. But we must sacrifice the smoothness of $W$ even if $V$ is smooth.

(5.4) As for the case $g=1$, we have the following

TheOREM. Let $L$ be an ample line bundle on a normal variety $V$ with $n=\operatorname{dim} V$ and suppose that $g(V, L)=1$ and $L$ is spanned by global sections. Then one of the following conditions are satisfied:

1) $V$ has only rational Gorenstein singularities, $\Delta(V, L)=1$ and $\omega=(1-n) L$ in Pic $(V)$ for the canonical sheaf $\omega$.

2) $(V, L)$ is a scroll over an elliptic curve, or a generalized cone over such a scroll.

(5.5) By "generalized cone" we mean the following: Let $\mathscr{A}$ be an ample vector bundle on an elliptic curve $C$ and set $\mathscr{E}=\mathcal{O}_{C}^{\oplus \kappa} \oplus \mathscr{A}, M=\boldsymbol{P}_{C}(\mathscr{E})$ and $H=\mathcal{O}_{M}(1)$. The surjection $\mathscr{E} \rightarrow \mathcal{O}_{C}^{\oplus k}$ defines a submanifold $N$ of $M$ such that $N \simeq \boldsymbol{P}\left(\mathcal{C}_{C}^{\oplus k}\right) \simeq C \times \boldsymbol{P}^{k-1}$ with $H_{N}$ being the pull-back of $\mathcal{O}(1)$ of $\boldsymbol{P}^{k-1}$. The linear system $|m H|$ has no base point for $m \gg 0$ and gives a birational morphism $\pi: M \rightarrow W$ onto a normal variety $W$ such that $H=$ $\pi^{*} A$ for some ample line bundle $A$ on $W$. Moreover $M-N \simeq W-\pi(N)$ and $\pi_{N}: N \rightarrow \pi(N)$ is the second projection of $N$ onto $P^{k-1}$. If $(V, L)$ is isomorphic to such a polarized variety $(W, A)$, it is called a generalized 
cone over $(\boldsymbol{P}(\mathscr{A}), \mathcal{O}(1))$. We remark that $W$ has irrational singularities along $\pi(N)$. They are not even Cohen Macaulay in general.

(5.6) We prove (5.4) by induction on $n$. The assertion is obvious when $n=1$, and is known when $n=2$ (cf. [Sa]). So we assume $n \geqq 3$. But the present method works also for $n=2$.

Let $\pi: M \rightarrow V$ be a desingularization of $V$, let $D$ be a general member of $|L|$ and set $S=\pi^{*} D \in\left|L_{M}\right|$. Then $D$ is normal and $S$ is smooth by Bertini type theorems. Since $g\left(D, L_{D}\right)=1$, we can apply induction hypothesis to $\left(D, L_{D}\right)$. If $\left(D, L_{D}\right)$ is of the type 1$)$, then $h^{1}(D, \mathcal{O})=h^{1}(S, \mathcal{O})=0$ by (3.2), since rational Gorenstein singularities are canonical. We have $h^{1}(S, \mathcal{O})=1$ if $\left(D, L_{D}\right)$ is of the type 2$)$.

Suppose that $h^{1}(M, \mathcal{O})=0$. Then $h^{1}(S, \mathcal{O})=0$ since $h^{2}\left(M,-L_{M}\right)=0$ by duality and (2.1). So $\left(D, L_{D}\right)$ is of the type 1). Hence $h^{0}\left(S, K^{s}+\right.$ $\left.(n-2) L_{S}\right)>0$ for the canonical bundle $K^{S}$ of $S$. This implies $h^{0}(M, K+$ $\left.(n-1) L_{M}\right)>0$ for the canonical bundle $K$ of $M$, since $h^{1}\left(M, K+(n-2) L_{M}\right)$ $=0$ by (2.1). Let $E$ be a member of $\left|K+(n-1) L_{M}\right|$. Then $L^{n-1} E=0$ since $g\left(M, L_{M}\right)=g(V, L)=1$ by (1.8). So $\operatorname{dim} \pi(E)<n-1$. Since $K=$ $(1-n) L_{M}+E$ in $\operatorname{Pic}(M)$, this implies that $V$ has only canonical singularities and $\omega_{V}=(1-n) L$. We have also $\Delta(V, L)=\Delta\left(M, L_{M}\right)=\Delta\left(S, L_{S}\right)$ $=\Delta\left(D, L_{D}\right)=1$. Thus $(V, L)$ is of the type 1$)$.

Suppose that $h^{1}(M, \mathcal{O})>0$. We have $h^{1}(M, \mathcal{O}) \leqq h^{1}(S, \mathcal{O})$ since $h^{1}\left(M,-L_{M}\right)=0$. So $1=h^{1}(M, \mathcal{O})=h^{1}(S, \mathcal{O})$ and $\left(D, L_{D}\right)$ is of the type 2). Let $\alpha: M \rightarrow C$ be the Albanese map. Clearly $C$ is an elliptic curve. Moreover, using the Stein factorization and the universality of the Albanese map, we infer that any general fiber $X$ of $\alpha$ is connected (cf. [U; p. 112]). Since $L_{X}$ is nef and big, $X \cap S$ is connected too. This implies $\operatorname{Alb}(S) \simeq C$ and the restriction $\sigma$ of $\alpha$ to $S$ is the Albanese map of $S$. Recall that $\left(S, L_{S}\right)$ is birationally equivalent to a scroll over $C$. So $\mathscr{F}=$ $\sigma_{*} \mathcal{O}_{S}\left(L_{S}\right)$ is locally free and the natural homomorphism $\sigma^{*} \mathscr{F} \rightarrow \mathcal{O}_{S}\left(L_{S}\right)$ is surjective. On the other hand, $h^{1}(X, \mathcal{O}) \leqq h^{1}(X \cap S, \mathcal{O})=0$ since $h^{1}\left(X,-L_{X}\right)$ $=0$ by (2.1). So $\mathscr{F}_{1}=R^{1} \alpha_{*} \mathcal{O}_{M}$ is supported in a finite subset of $C$. Moreover, using Leray spectral sequence, we infer $h^{0}\left(C, \mathscr{F}_{1}\right)=0$ since $h^{1}(M, \mathcal{O})=h^{1}(C, \mathcal{O})$. So $\mathscr{F}_{1}=0$. Now, taking $\alpha_{*}$ of $0 \rightarrow \mathcal{O}_{M} \rightarrow \mathcal{O}_{M}\left(L_{M}\right) \rightarrow$ $\mathcal{O}_{S}\left(L_{S}\right) \rightarrow 0$, we get an exact sequence $0 \rightarrow \mathcal{O}_{C} \rightarrow \mathscr{E} \rightarrow \mathscr{F} \rightarrow 0$ for $\mathscr{E}=\alpha_{*} \mathcal{O}_{M}\left(L_{M}\right)$. So $\mathscr{E}$ is locally free. Moreover, we easily see that $\alpha^{*} \mathscr{E} \rightarrow \mathcal{O}_{M}\left(L_{M}\right)$ is surjective. Hence there is a morphism $f: M \rightarrow P=\boldsymbol{P}(\mathscr{E})$ such that $L_{M}=$ $f^{*} \mathcal{O}_{P}(1)$. 
Suppose in addition that $\alpha$ factors through $V$. Let $\beta: V \rightarrow C$ be the morphism such that $\alpha=\beta \circ \pi$. Then $\mathscr{E}=\beta_{*} \mathcal{O}_{V}(L)$ since $V$ is normal. Moreover $\beta^{*} \mathscr{E} \rightarrow \mathcal{O}_{V}(L)$ is surjective. So we have $\varphi: V \rightarrow P$ such that $L=\varphi^{*} \mathcal{O}_{P}(1) . \varphi$ is finite since $L$ is ample. Hence it is an isomorphism by Zariski's Main Theorem. Thus $(V, L)$ is a scroll over $C$.

If $\alpha$ does not factor, then there is a curve $Y$ in $M$ such that $y=\pi(Y)$ is a point while $\alpha(Y)=C$. We may assume $y \notin D$ since $D$ is a general member of $|L|$. By construction of $f$, there is a divisor $H$ in $P$ such that $f^{*} H=S$ and $H \simeq \boldsymbol{P}(\mathscr{F})$. Since $y \notin D$ and $Y \cap S=\varnothing$, we have $f(Y) \cap H$ $=\varnothing$. Therefore, the pull-back of the exact sequence $0 \rightarrow \mathcal{O}_{C} \rightarrow \mathscr{E} \rightarrow \mathscr{F} \rightarrow 0$ via the map $Y \rightarrow C$ splits on $Y$. So this sequence splits on $C$ since $H^{1}(C, \mathscr{F} \vee) \rightarrow H^{1}(Y, \mathscr{F} \vee)$ is injective. By the induction hypothesis we have $\mathscr{F} \simeq \mathcal{O}_{C}^{\oplus k} \oplus \mathscr{A}$ for some $k \geqq 0$ and an ample vector bundle $\mathscr{A}$ on $C$. So $\mathscr{E} \simeq \mathcal{O}^{\oplus(k+1)} \oplus \mathscr{A}$. From this we infer that $(V, L)$ is birationally equivalent to a generalized cone over $C$. But they must be isomorphic since $L$ is ample.

(5.7) In order to proceed further, we want to study $\omega+m L$ for general $m>0$. The result [S2; (2.1)] is very useful for this purpose. The following theorem improves upon this result slightly.

Theorem. Let $(V, L)$ be a polarized variety and let $A$ be an irreducible reduced member of $|L|$. Suppose that

1) the double dual $\omega^{r}$ of the $r$-th tensor product of the canonical sheaf $\omega$ of $V$ is invertible for some $r>0$, and $\omega^{m}$ is invertible in a neighborhood of $A$ for a possibly smaller positive integer $m$,

2) $U=V-Y$ has only log-terminal singularities (for $B=0$ ) for some finite set $Y$, and

3) $(\omega+t L)_{A}$ is a nef $\boldsymbol{Q}$-bundle on $A$ for some rational number $t$ with $t \geqq 2-m^{-1}$.

Then $\omega+t L$ is nef on $V$ unless $n=\operatorname{dim} V=2$ and $(V, L)$ is a scroll over a curve isomorphic to $A$.

Proof. Set $\sigma=\inf \{s \in Q \mid \omega+s L$ is nef on $V\}$. We study the case $\sigma>t$.

By [F7; (6.10)], there exists $k>0$ such that the restriction of $\mathscr{F}(a, b)$ $=\omega^{a m} \otimes \mathcal{O}(b L)$ to $A$ is very ample for any positive integers $a, b$ with $b>a m t+k$. We claim that there is such a pair $(a, b)$ with $b<a m \sigma$ and $b-1 \geqq(a m-1) \sigma$. 
Indeed, if $a \gg 0$ and $b$ is the largest integer with $b<a m \sigma$, then $b>a m t+k$ since $\sigma>t$. Moreover, if $\sigma \notin \boldsymbol{Q}$, then $a m \sigma-b$ can become arbitrarily small for a suitable $a$, so we have $b-1 \geqq(a m-1)_{\sigma}$ too. When $\sigma \in \boldsymbol{Q}$, we set $m \sigma=q / p$ for some mutually coprime integers $p, q$. Then $a m \sigma-b=1 / p$ for a suitable $a \gg 0$. Since $\sigma>2-m^{-1}$, we have $\sigma \geqq 1+1 / p$, which implies $b-1 \geqq(a m-1) \sigma$ as desired.

Set $\mathscr{F}=\mathscr{F}(a, b)$ for $a, b$ as above. Similarly as in (3.2), we have $H^{1}(V, \mathscr{F}(-L))=0$ unless $b-1=(a m-1) \sigma$ and $\omega+\sigma L$ is not big. The vanishing of $H^{1}$ implies that $H^{0}(V, \mathscr{F}) \rightarrow H^{0}\left(A, \mathscr{F}_{A}\right)$ is surjective. So $\mathscr{F}$ is spanned by global sections in a neighborhood of $A$, hence so is $\mathscr{F}^{r}$, which is invertible on $V$. Therefore $\mathrm{Bs}\left|\mathscr{F}^{r}\right|$ is finite since $A$ is ample. Then $\mathscr{F}$ is nef, which contradicts $b<a m \sigma$.

Now we conclude that $b-1=(a m-1) \sigma$ and $\omega+\sigma L$ is not big. Take a large integer $l$ such that $l \sigma \in Z, l \sigma>l t+k$ and $\omega^{l}$ is invertible on $V$. Then, similarly as above, $\mathrm{Bs}|F|$ is finite for $F=\omega^{l}\left(l_{\sigma} L\right)$. By [F7; (1.14)] $F$ is semiample. So there is a surjective morphism $f: V \rightarrow W$ onto a normal variety $W$ such that $F=f^{*} H$ for some ample $\boldsymbol{Q}$-bundle $H$ on $W$. Note that $\operatorname{dim} W<n=\operatorname{dim} V$ since $\omega+\sigma L$ is not big.

We claim that the restriction $f_{A}: A \rightarrow W$ of $f$ is a finite morphism. Indeed, otherwise, there is a curve $C$ in $A$ such that $(\omega+\sigma L) C=0$. But $(\omega+t L) C \geqq 0$ since $C \subset A$. This contradicts $\sigma>t$.

This claim implies $\operatorname{dim} W^{\top}=n-1$. Moreover any general fiber $Z$ of $f$ is a normal curve with $(\omega+\sigma L) Z=0$. So $\omega Z<0$ and $Z \simeq \boldsymbol{P}^{1}$. Hence $\omega Z=-2, L Z=1$ and $\sigma=2$ since $\sigma>2-m^{-1} \geqq 1$. Therefore $f_{A}$ is birational. So $A \simeq W$ by Zariski's Main Theorem.

Now we claim $n=2$. Indeed, otherwise, the restriction map $\operatorname{Pic}(V)$ $\rightarrow \operatorname{Pic}(A)$ is injective by [F2; (2.6)]. This implies $L=f^{*}\left(f_{A}^{-1}\right)^{*} L_{A}$, yielding a contradiction as in [S1].

Thus $W$ is a curve and every fiber $X$ of $f$ is a Cartier divisor with $L X=L Z=1$. From this we infer that $(V, L)$ is a scroll over $W$ by an argument in (3.7).

Q.E.D.

Remark. [S2; (2.1)] takes care the case $m=1$ and $t \geqq 2$.

(5.8) Using the above theorem, we can generalize many results which follow from [S2; (2.1)]. We have also the following

Corollary. Let things be as above and suppose that $A$ is locally Gorenstein and that the canonical sheaf of $A$ is nef. Then $\omega+L$ is nef 
on $V$ unless $A$ is a curve and $(V, L)$ is a scroll over $A$.

Proof. Apply (5.7) for $m=t=1$.

Note added in proof. After this paper was written, I found that (2.6) was proved by T. Luo in Amer. J. Math., 111 (1989), 457-487; Theorem 2.1 by a very different method. Further, very recently, he gave a new simpler proof which works in positive characteristic cases too (A Note on the Hodge Index Theorem, preprint, 1989).

\section{REFERENCES}

[F1] T. Fujita, On the structure of polarized varieties with 4 -genera zero, J. Fac. Sci. Univ. of Tokyo, 22 (1975), 103-115.

[F2] _- On the hyperplane section principle of Lefschetz, J. Math. Soc. Japan, 32 (1980), 153-169.

[F3] - On the structure of polarized manifolds with total deficiency one, part I, II \& III, J. Math. Soc. Japan, 32 (1980), 709-725, 33 (1981), 415-434 \& 36 (1984), 75-89.

[F4] - On $L$-dimension of coherent sheaves (with correction), J. Fac. Sci. Univ. of Tokyo, 28 (1981), 215-236 \& 29 (1982), 719-720.

[F5] - On polarized varieties of small $\Delta$-genera, Tôhoku Math. J., 34 (1982), 319-341.

[F6] - Theorems of Bertini type for certain types of polarized manifolds, J. Math. Soc. Japan, 34 (1982), 709-718.

[F7] - Semipositive line bundles, J. Fac. Sci. Univ. of Tokyo, 30 (1983), 353-378.

[F8] Z Zariski decomposition and canonical rings of elliptic threefolds, J. Math. Soc. Japan, 38 (1986), 19-37.

[F9] _- Projective varieties of $\Delta$-genus one, in Algebraic and Topological Theories -to the memory of Dr. Takehiko MIYATA, pp.149-175, Kinokuniya, Tokyo, 1985.

[F10] - On polarized manifolds whose adjoint bundles are not semipositive, in Algebraic Geometry Sendai 1985, pp. 167-178, Advanced Studies in Pure Math. 10, Kinokuniya, 1987.

[F11] - Notes on quasi-polarized varieties, Proc. Japan Acad., 64 (1988), 88-90.

[H] H. Hironaka, Resolution of singularities of an algebraic variety over a field of characteristic zero, Ann of Math., 79 (1964), 109-326.

[I] P. Ionescu, Generalized adjunction and applications, Math. Proc. Cambridge Phil. Soc., 99 (1986), 457-472.

[K] Y. Kawamata, A generalization of Kodaira-Ramanujam's vanishing theorem, Math. Ann., 261 (1982), 43-46.

[KMM] Y. Kawamata, K. Matsuda and K. Matsuki, Introduction to the minimal model problem, in Algebraic Geometry Sendai 1985, pp. 283-360, Advanced Studies in Pure Math. 10, Kinokuniya, 1987.

[M1] S. Mori, Threefolds whose canonical bundles are not numerically effective, Ann. Math., 116 (1982), 133-176.

[M2] - Flip theorem and the existence of minimal models for threefolds, J. of AMS, 1 (1988), 117-253. 
[R] M. Reid, Canonical 3-folds, in Géométrie Algébrique Angers 1979 pp. 273-310, Sijthoff \& Noordhoff, Alphen aan den Rijn, 1980.

[Sa] F. Sakai, Ample Cartier divisors on normal surfaces, J. reine angew. Math., 366 (1986), 121-128.

[Sh] V. V. Shokurov, Theorem on non-vanishing, Math. USSR-Izv., 26 (1986), 591604 .

[S1] A. J. Sommese, On manifolds that cannot be ample divisors, Math. Ann., 221 (1976), 55-72.

[S2] - On the adjunction theoretic structure of projective varieties, in Complex Analysis and Algebraic Geometry, pp. 175-213, Lecture Notes in 1194, Springer, 1986.

[U] K. Ueno, Classification Theory of Algebraic Varieties and Compact Complex Spaces, Lecture Notes in Math. 439, Springer, 1975.

[V] E. Viehweg, Vanishing theorems, J. reine angew. Math., 335 (1982), 1-8.

Department of Mathematics

College of Arts and Sciences

University of Tokyo

Komaba, Meguro, Tokyo 153

Japan 